\title{
Safety, cost and effectiveness issues with disease modifying anti-rheumatic drugs in rheumatoid arthritis
}

\author{
James F Fries
}

Safety, cost, and effectiveness studies are critically important for definitive assessment of disease modifying drugs (DMARDs), particularly when new mechanisms of drug action are likely to be present. This requires post-marketing surveillance, as these dimensions cannot be adequately assessed in pre-marketing studies. "Post-marketing surveillance", in our definition, is "the asking and answering of important questions the answers to which cannot be ascertained from premarketing trials". ${ }^{1}$ Such questions include assessing the long term toxicity of the new drugs, comparison of the toxicity of the new drugs with alternative treatments, assessment of toxicity in elderly populations and persons with comorbid diseases, noting the characteristics of patients likely to experience toxicity, exploring the effects of drug combinations on efficacy and toxicity, and evaluating the long term net cost of treatment.

For each of these questions, long term data are required. Such studies may require 1000 to 10000 patient years of experience for each drug treatment arm. Randomised trials of this scope are not practical. Adverse event reporting systems lack accurate numerators and denominators and cannot validly compare different agents. Hence, prospective, protocol driven, large scale, observational studies are required. These should be started as early in the drug development sequence as possible. Data analysis must be sophisticated and must go far beyond purely descriptive studies without controls. Often, enrollment of early patients in phase 2 and 3 may shorten the time required for adequate follow up.

The chronic disease data bank model ARAMIS (The Arthritis, Rheumatism, and Aging Medical Information System) is the archetype of a chronic disease data bank. ${ }^{2}$ Over the past 23 years and through over 1000 publications, ARAMIS has used large scale data sets, lifetime follow up, and defined populations. Consecutive patients at each of multiple centres are enrolled and followed up for life. Data collected include clinical information such as that in the medical record, as well as protocol driven collection of effectiveness, costs, mortality, and safety data, at six month intervals, using the Health Assessment Questionnaire (HAQ). Hospitalisations are audited through discharge summaries and deaths through the National Health Index and death certificates. Sufficient data are accumulated to establish background frequencies of serious events, ${ }^{3}$ to allow comparative data on alterna- tive treatments, ${ }^{4}$ to use the Toxicity Index as an approach to comparisons of safety in agents with different side effect profiles, ${ }^{56}$ and to provide strong and experienced methodological support. We believe that long term surveillance needs to include the several outcome dimensions of interest, including mortality and its causes, hospitalisations and their causes, use of medical care services, and long term effects on cumulative disability and cumulative costs. ${ }^{78}$

ARAMIS rheumatoid arthritis programmes include eight data bank centres including university clinics, private practices, and community samples: Stanford, Wichita, Baltimore, Cincinnati, Phoenix, Santa Clara County, Saskatchewan, and Montreal. Data on 6673 RA patients with a total of 51241 years of follow up currently are available. The 3883 RA patients with 19691 years of HAQ follow up constitute the major population. Additionally, data on 5193 osteoarthritis patients with a total of 19692 patient years of follow up are available. $^{9}$

Outcome models for rheumatoid arthritis A multi-factorial outcome model for rheumatoid arthritis is shown in figure $1 .^{10}$ Patients, with particular genetic characteristics, experience an external perturbation, usually in mid-life, resulting in inflammatory pannus forming in and around the joints. The intensity of the inflammation and the duration of the inflammation combine to digest articular and periarticular structures slowly over time and may lead to adverse outcomes of disability, premature mortality, and large medical expenses. The basic medical therapeutic approach is to reduce the amount of inflammation and the duration of inflammation, hence limiting the autodigestion of tissues. A necessary requirement is the optimal sequencing of therapeutic agents over a disease course averaging 25 years in length. There may be failure to start definitive treatment early or to use major therapy only intermittently, and thus to leave progressive disease in place. Side effects of treatment may effect the ability to sequence treatments optimally, may prevent the use of some potentially useful drugs, and may contribute directly to adverse outcomes. Non-biological factors such as the presence of risk factors for comorbid diseases, lack of exercise, and poor personal self efficacy also may contribute to poor outcomes. Hence, the complex multi-factorial model includes patient factors, disease factors, treatment factors, and the lifestyle responses of the individual patient to a chronic illness. 


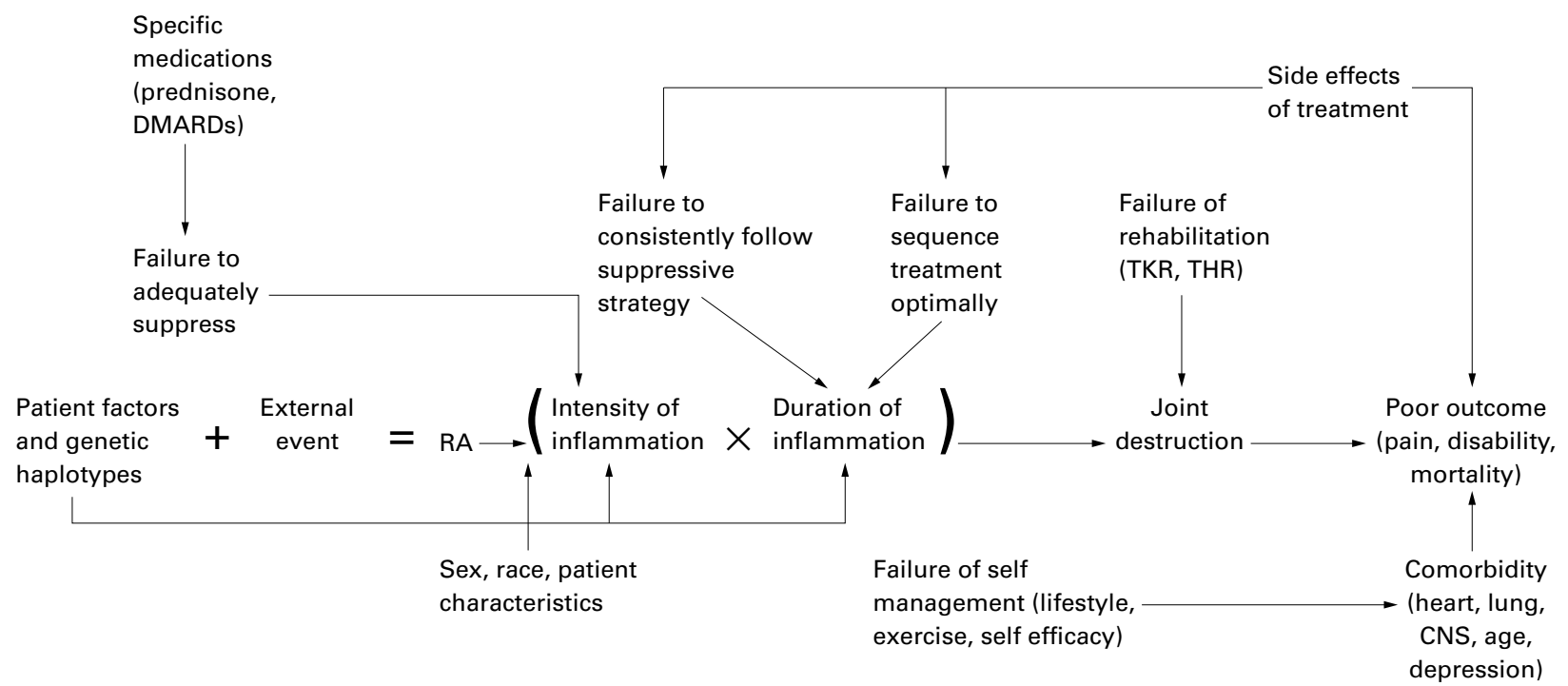

Figure 1 A multifactorial model showing inputs important to long term outcomes in RA.

The special problems of DMARDs

DMARDs are the basis of effective treatment of RA. By definition, they must modify the disease course favourably, including retarding effects on bony and structural elements. They must limit the long term morbidity of the disease. As a drug category (although not necessarily as single agents) they must decrease cumulative disability over 25 years. If successful, they might also decrease the standardised mortality rate of RA from its current level of 2 to 3 to 1 toward 1 , therefore being themselves life extending.

Long term safety studies in RA must deal with delayed problems, such as neoplasia, osteopenia, infections, and autoimmune disease, requiring prospective long term study to determine incidence rates. The background frequencies of serious events are often increased in RA, including lymphoma, infection, gastrointestinal events, and transition to other autoimmune states. The toxicities of alternative treatments need to be subtracted from the toxicities of the study drugs to estimate net effects. The degree of comparative effectiveness of alternative drugs also enters the equation. From the social perspective, the cost of treatment requires consideration.

\section{Net cost and net effectiveness}

\begin{tabular}{ll} 
Costs: & Effectiveness: \\
Direct medical costs & Decreased disability, pain \\
Cost of side effects & Decreased mortality rates \\
Cost of long term side effects & Decreased global health \\
\multicolumn{1}{c}{-minus- } & \multicolumn{1}{c}{-minus- } \\
Avoided surgery & Drug related symptoms \\
Avoided long term care & Drug related disability \\
Avoided hospitalisations & Drug related mortality \\
Continued employment & Drug related absenteeism
\end{tabular}

Continued employment

Drug related absenteeism

Figure 2 Costs versus effectiveness and the offsets that can be found either on the cost and adverse effect side or on the effectiveness side.
Counting all costs, all toxicity, and all effectiveness, over time

These problems are formidable but are beginning to yield to new assessment techniques. The major lesson of recent years with regard to the costs of medical care is that all costs must be counted. It is not just the cost of the medication but the extra medical visits, the laboratory tests, the costs of treating side effects, the comorbid therapy given to prevent side effects, and so forth. The same lesson applies to effectiveness and to side effects. Effectiveness assessment must include the right variables and must include both early and late effects. Side effects assessment must include serious delayed problems as well as the more easily measured acute effects. Less frequently recognised (fig 2) is that there can be offsets either on the cost and adverse effect side or on the effectiveness side.

From direct costs must be subtracted the cost savings from surgery that might be averted, potential decreases in the need for expensive long term care, prevented hospitalisations, and continued employment. Long term effectiveness is directed at decreasing cumulative disability and pain, potentially decreasing disease related mortality rates, and improving patient global health, all integrated over time. But from the effectiveness data must be subtracted any drug related symptoms, any drug related disability, any drug related mortality, and any drug related absenteeism from employment, also considered overtime.

\section{Potential solutions}

This is clearly an area of difficult analyses, but some emerging solutions offer help. The central problem is the availability of long term data with the appropriate end points included. As described above, the Chronic Disease Data Bank can provide data developed from consistent protocols on alternative drugs, and can establish the baseline frequencies of adverse events caused by the disease process itself or from externalities and not the treatment. The ability to count all of the costs and all of the 




Figure 3 The relation between current disability levels and medical care costs over the next five years.

effectiveness measures over a long time period gives insights, and data from studies of two to five years duration may be used to project future costs and side effects.

The measurement of disability is the key to prediction of future costs. Indeed, in predicting costs over the next $5-10$ years in RA patients, disability (and the squared term of disability) dominate all other measures and explain most of the variance that may be explained. ${ }^{11}$ Age, male sex, disease duration, and education level are also important but of much smaller magnitude. Indeed, in ARAMIS data, once disability is entered into a model, no other variables are statistically significant. Future costs are not well predicted by specific clinical observations at one point in time; but may be estimated from disability levels.

Figure 3 shows the relation between current disability level and medical care costs over the following five years. The association is strong and progressive. This should not be surprising, as it is disability that clearly drives the major cost items, including hospitalisation, surgery, loss of employment, and need for long term care. Thus, it is possible, given a new treatment with substantial reduction in disability after two to five years, to make reasonable projec-

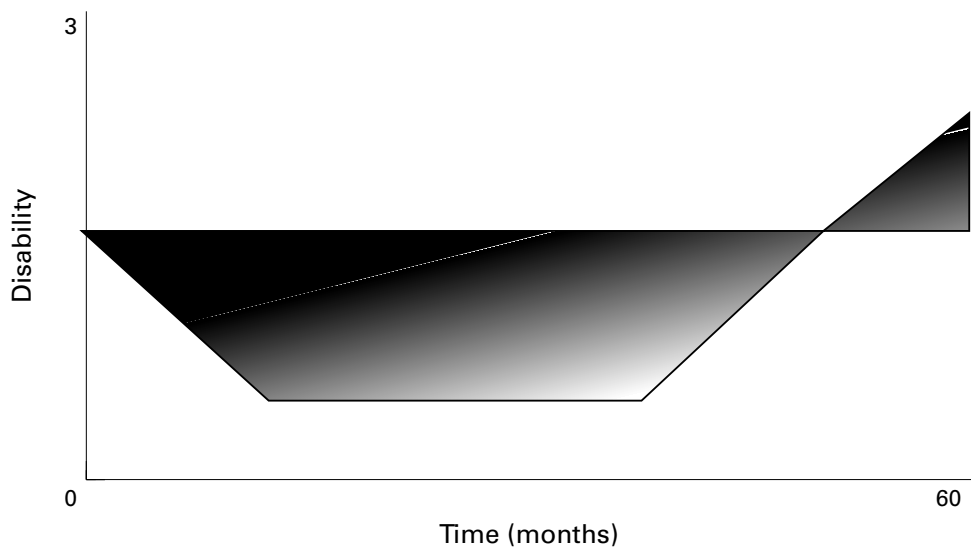

Figure 4 The therapeutic segment. Effectiveness over the period of the segment may be computed as the disability years below the initial disability level minus the disability years above that level. tions about effects on future disability (predicted primarily by present disability) and future costs.

The concept of the therapeutic segment Effective long term treatment in RA may be considered as a problem in drug sequencing. Some sequences will be more effective than others, and different sequences may be optimal in different patient groups. Over the 25 year course of RA, a number of drugs, alone and in combination, are nearly always used. The period from the beginning of a new agent to the time of the next treatment change may be termed the "therapeutic segment". ${ }^{11}$ The therapeutic segment (fig 4) has a different expected duration for each drug, with methotrexate currently representing the longest segment, approximately five years in the typical patient. The therapeutic segment also has a level of effectiveness, a point to maximum benefit, a point beyond which benefit is lost, and may be represented as the area under the curve as shown in figure 4 . Beyond this, there are patient, disease, and sequencing factors that enable the prognosis for a therapeutic segment for a given patient to be predicted more accurately. New therapeutic agents, with their own therapeutic segment characteristics, are certain to become available and to change the optimal drug sequence.

If the course of RA is visualised as a series of therapeutic segments; knowledge of the different effectiveness of segments representing different drugs and different drug combinations allows decision analytic techniques to be used to optimise drug sequencing. When a new DMARD is introduced, the clinical question becomes where in the sequencing of therapeutic segments it is most appropriately used. Depending upon the characteristics of the therapeutic segment of the new drug, it could find a role in early disease, in mid-disease, or in late disease, in those with particularly aggressive disease, in those with relatively minor problems, and so forth. From this perspective it becomes important to define the characteris- 
tics of a therapeutic segment for new therapeutic agents and to compare these characteristics as soon as possible with the therapeutic segments of established drugs that have had a longer clinical experience.

\section{Improving long term outcomes in RA}

The optimal sequence of treatment of RA in terms of reducing lifetime morbidity, mortality, and perhaps medical costs is the central issue in RA management. Classically, the sequencing of medications and the correct use of new agents, has not been driven by data; witness the old "treatment pyramid", created by good intentions and faulty premises. With emerging techniques and new perspectives we may move relatively rapidly to an era in which experience with new agents feeds back promptly into improved sequencing of therapeutic agents. The goal of improving long term outcome requires a long term perspective. Early modelling by the ARAMIS group suggests that use of the most effective DMARD earlier in the course is likely to have greater effects upon long term outcomes. ${ }^{12}$ Thus, particularly in some patient subgroups, there may be need for very aggressive approaches to early disease. A conceptual problem is how to include savings in long term morbidity and costs in terms of fewer hospitalisations, less nursing home care, greater employment, and reduced mortality into models as offsets for early toxicity and early costs.
Funding: this work was supported by a grant from the National Institutes of Health to ARAMIS (Arthritis, Rheumatism, and Aging Medical Information System) (AR43584).

1 Fries JF. The ARAMIS (American Rheumatism Association Medical Information System) Post-Marketing Surveillance Program. Drug Information Journal 1985;19:257-62.

2 Fries JF. The chronic disease data bank model: A conceptual framework for the computerbased medical record. Comp Biomed Res 1992;25:586-601.

3 Williams CA, Bloch DA, Sibley J, et al. Lymphoma and leukemia in rheumatoid arthritis: Are they associated with azathioprine, cyclophosphamide, or methotrexate? A matched case-control study in the Arthritis, Rheumatism and Aging Medical Information System (ARAMIS) population. J Clin Rheumatol 1996;2:64-72.

4 Fries JF, Williams CA, Bloch DA. The relative toxicity of non-steroidal anti-inflammatory drugs (NSAIDs). Arthritis Rheum 1991;34:1353-60.

5 Fries JF, Spitz PW, Williams CA, Bloch DA, Singh G, Hubert HB. A toxicity index for comparison of side effects among different drugs. Arthritis Rheum 1990;33:121-30.

6 Fries JF, Williams CA, Ramey D, Bloch DA. The relative toxicity of disease modifying antirheumatic drugs (DMARDs). Arthritis Rheum 1993;36:297-306.

7 Fries JF. Toward an understanding of patient outcome measurement. Arthritis Rheum 1983;26:697-704.

8 Ramey DR, Fries JF, Singh G. The Health Assessment Questionnaire 1995 - Status and Review. 2nd ed. Spilker B, ed. Pharmacoeconomics and quality of life in clinical trials. New York: Raven Press, 1996;227-37.

9 Fries JIF. Non-steroidal anti-inflammatory drug safety: a view from the ARAMIS databank. Curr Opin Rheumatol 1996;9:S3-8.

10 Fries JF. Effectiveness and toxicity considerations in outcome-directed therapy in rheumatoid arthritis. J Rheumatol 1996;23:102-6.

11 Fries JF, Williams CA, Ramey DR, Singh G. Medical costs are strongly associated with disability levels in rheumatoid arthritis (RA). Arthritis Rheum 1995;38:S187.

12 Albert DA, Aksentijevich S, Hurst S, Fries JF, Wolfe F. Modeling therapeutic strategies in rheumatoid arthritis: Use of decision analysis and Markov models. Arthritis Rheum 1998;41:S227. 